\title{
OSTEOTOMIAS SEGMENTARES MÚLTIPLAS PARA A CORREÇÃO DA CIFOSE
}

\author{
MULTIPLE SEGMENTAL OSTEOTOMIES TO \\ THE KYPHOSIS CORRECTION
}

\begin{abstract}
Carlos Fernando Pereira da Silva Herrero', Maximiliano Aguiar Porto', Marcello Henrique Nogueira Barbosa², Helton Luiz Aparecido Defino ${ }^{3}$
\end{abstract}

\section{RESUMO}

Objetivo: Avaliar o resultado do tratamento cirúrgico da hipercifose dorsal da coluna vertebral por meio da técnica de Ponte (osteotomias múltiplas posteriores). Métodos: Estudo retrospectivo de 10 pacientes (oito com sequela de doença de Scheuermann e dois com sequela de laminectomia) submetidos a cirurgia para correção de hipercifose acima de $70^{\circ}$. A idade variou de 12 anos a 20 anos (média de 16,8 anos $\pm 2,89$ ). Os parâmetros radiográficos estudados foram a mensuração da cifose, lordose e, quando presente, da escoliose. Também foram avaliadas a presença de cifose juncional proximal e distal, a perda da correção e complicações como soltura e quebra dos implantes. Os parâmetros radiográficos foram avaliados no período pré-operatório, pós-operatório imediato e avaliação tardia. Resultados: Os pacientes foram seguidos por um período que variou de 24 a 144 meses (média de 65,8 meses $\pm 39,92$ ). O valor médio da hipercifose pré-operatória foi de $78,8^{\circ} \pm 7,59^{\circ}$ (Cobb) e de $47,5^{\circ} \pm 12,54^{\circ}$ no seguimento, com a média de correção de $33,9^{\circ} \pm 9,53^{\circ}$ e perda média de correção de $2,2^{\circ}$. Conclusão: $\mathrm{O}$ tratamento cirúrgico da hipercifose torácica por meio de osteotomias múltiplas posteriores apresentou boa correção da deformidade e perda mínima de correção ao longo do seguimento.

Descritores - Cifose; Coluna vertebral; Doença de Scheuermann; Fusão vertebral; Osteotomia

\section{ABSTRACT}

Objective: To evaluate the results of the surgical treatment of the spinal Kyphosis using the Ponte's technique (multiple posterior osteotomies). Methods: Ten patients ( 8 with Scheuermann's kyphosis and 2 with kyphosis after laminectomy) submitted to surgical correction of kyphotic deformity greater than $70^{\circ}$ were retrospectively assessed. The age at the surgical time ranged from 12 to 20 years old (mean age 16.8 years \pm 2.89 ). The radiographic parameters evaluated were the kyphosis, the lordosis and the scoliosis - whenever present. The presence of proximal and distal junctional kyphosis, loss of correction, and complications as implants loosening and breakage were also assessed. The radiographic parameters were evaluated at the preoperative, early postoperative and late postoperative time. Results: The patients were followed through a period that ranged from 24 to 144 months (65.8 \pm 39.92$)$. The mean value of the kyphosis was $78.8^{\circ} \pm$ $7.59^{\circ}$ (Cobb) before surgery and $47.5^{\circ} \pm 12.54^{\circ}$ at late follow up, with mean correction of $33.9^{\circ} \pm 9.53^{\circ}$ and lost correction of $2.2^{\circ}$. Conclusion: The surgical treatment of the thoracic kyphosis using multiples posterior osteotomies presented a good correction of the deformity and minimal lost of correction during follow up.

Keywords - Kyphosis; Spine; Scheuermann disease; Spinal fusion; Osteotomy

1 - Pós-graduando do Departamento de Biomecânica, Medicina e Reabilitação do Aparelho Locomotor do Hospital das Clínicas da Faculdade de Medicina de Ribeirão Preto-USP - Ribeirão Preto (SP), Brasil.

2 - Professor Doutor da Divisão de Radiologia do Departamento de Clínica Médica da Faculdade Medicina de Ribeirão Preto-USP - Ribeirão Preto (SP), Brasil.

3 - Professor Titular do Departamento de Biomecânica, Medicina e Reabilitação do Aparelho Locomotor do Hospital das Clínicas da Faculdade de Medicina de Ribeirão Preto-USP - Ribeirão Preto (SP), Brasil.

Trabalho realizado no Hospital das Clínicas da Faculdade de Medicina de Ribeirão Preto-USP - Departamento de Biomecânica, Medicina e Reabilitação do Aparelho Locomotor - Ribeirão Preto (SP), Brasil.

Correspondência: Helton LA Defino. Av. Bandeirantes, 3.900, 11 and. - 14048-900 - Ribeirão Preto (SP), Brasil. E-mail: hladefin@fmrp.usp.br 


\section{INTRODUÇÃO}

O aumento da cifose fisiológica ou a redução da lordose é denominado de hipercifose e pode ocorrer ao longo de toda a coluna vertebral, sendo mais frequente ao nível da coluna torácica ${ }^{(1-3)}$. A etiologia da hipercifose inclui várias doenças, destacando-se pela sua frequência a doença de Scheuermann, as iatrogênicas e as póstraumáticas ${ }^{(4-6)}$

Os valores fisiológicos da cifose torácica apresentam grandes variações nos indivíduos, sendo considerados normais entre 20 e 40 graus $^{(7-9)}$. Na transição toracolombar a cifose acima de 20 graus é considerada patológica. A identificação do aumento da cifose pode ser mais difícil devido ao mecanismo de compensação dos segmentos proximais ou distais ${ }^{(9,10)}$.

O tratamento conservador da hipercifose dorsal tem indicação nos pacientes em crescimento e portadores da doença de Scheuermann com valores angulares abaixo de 70 graus $^{(1,11,12)}$. A indicação do tratamento cirúrgico independe da sua etiologia. Ele está indicado nos pacientes com cifose acima de 70 graus $^{(10-13)}$, acompanhada de dor, desequilíbrio sagital, queixas estéticas ou incapacidade funcional ${ }^{(13,14)}$.

O tratamento cirúrgico convencional é realizado por meio da abordagem anterior e posterior, com a finalidade de atender aos requisitos biomecânicos de restaurar o alinhamento sagital da coluna dorsal ${ }^{(15)}$. A abordagem anterior visa à liberação das estruturas e realização da artrodese intersomática, enquanto que a abordagem posterior corrige e estabiliza a deformidade por meio do encurtamento da sua superfície convexa ${ }^{(16-19)}$. Ponte e Siccardi ${ }^{(19)}$, para evitar a abordagem anterior e reduzir a morbidade do procedimento cirúrgico, desenvolveram a técnica da abordagem posterior isolada.

O objetivo do trabalho é apresentar o resultado clínico e radiológico da utilização da abordagem posterior isolada realizando osteotomias segmentares múltiplas da coluna vertebral para o tratamento da hipercifose dorsal.

\section{MÉTODOS}

Foram avaliados prontuários médicos de 10 pacientes (sete do sexo masculino e três do feminino) com idade entre 12 e 20 anos (média de 16,8 anos $\pm 2,89$ ). Oito pacientes apresentavam sequela da doença de Scheuermann e dois, sequela de laminectomia dorsal para remoção de tumor intrarraquídeo (astrocitoma pilocítico). Os dados gerais dos pacientes estão ilustrados na Tabela 1 .
A indicação do tratamento cirúrgico centrou-se no grau de deformidade e insatisfação com a cosmética. Todos os pacientes apresentavam cifose dorsal com valor angular acima de 70 graus. No período pré-operatório os pacientes foram submetidos à ressonância magnética da coluna vertebral dorsal para avaliar a integridade do canal medular; a identificação da redução do diâmetro do canal medular devida à protrusão discal é contraindicação para a realização da correção posterior da hipercifose dorsal $^{(20)}$.

Os pacientes foram submetidos ao tratamento cirúrgico de acordo com a técnica descrita por Ponte e Siccardi ${ }^{(19)}$ para a correção da hipercifose. Essa técnica consiste na realização de múltiplas osteotomias por meio da ressecção parcial bilateral das lâminas e facetas articulares vertebrais (Figuras 1 e 2). A correção e fixação do segmento vertebral afetado foram realizadas por meio da utilização de sistemas de fixação pediculares. Em quatro pacientes foi utilizado o sistema USIS-Ulrich, composto por hastes rosqueadas de $4 \mathrm{~mm}$, e em seis pacientes, o sistema USS-Synthes, composto por haste rígida de $6 \mathrm{~mm}$.

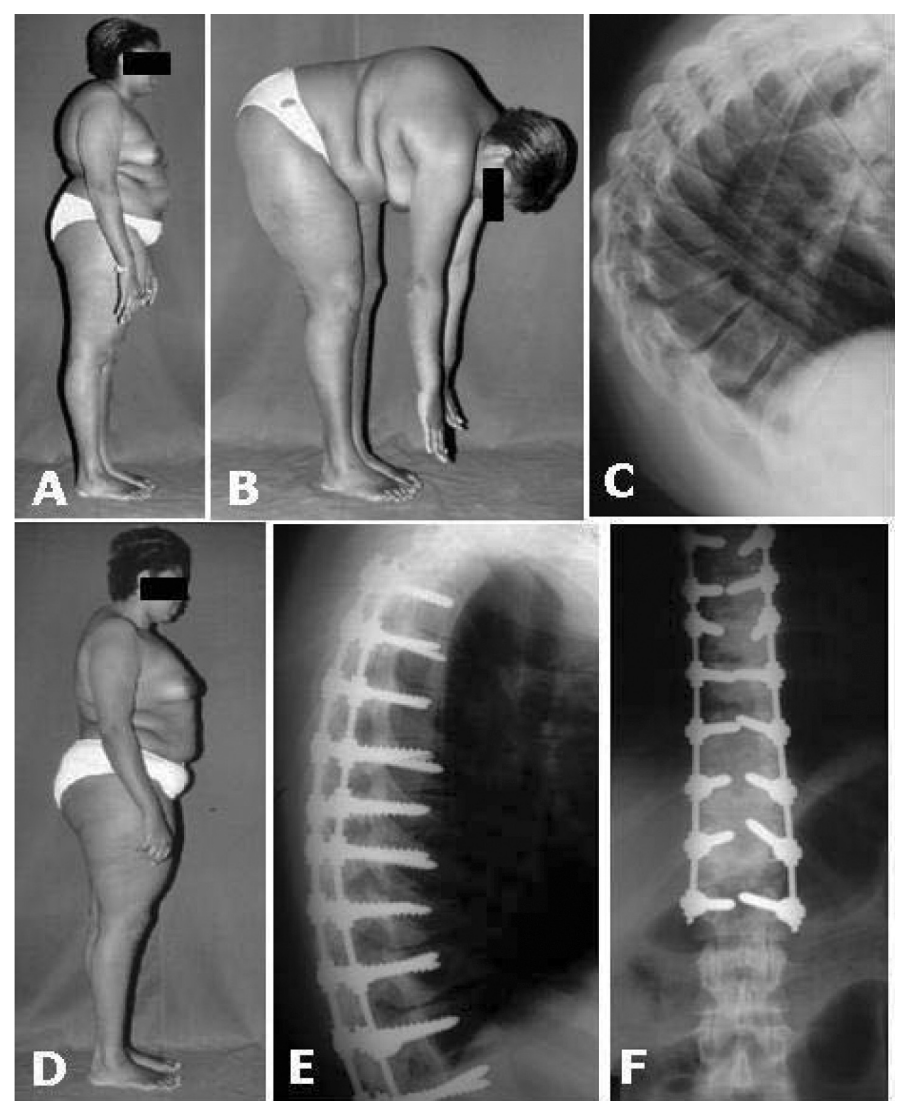

Figura 1 - Paciente no 7 da Tabela 1. A e B) Fotografias préoperatórias evidenciam aumento da cifose. $\mathrm{C}$ ) Incidência radiográfica em perfil evidencia hipercifose torácica. D) Imagem pósoperatória evidenciando correção da cifose. E e F) Incidências radiográficas em perfil e AP mostrando a correção da cifose. 


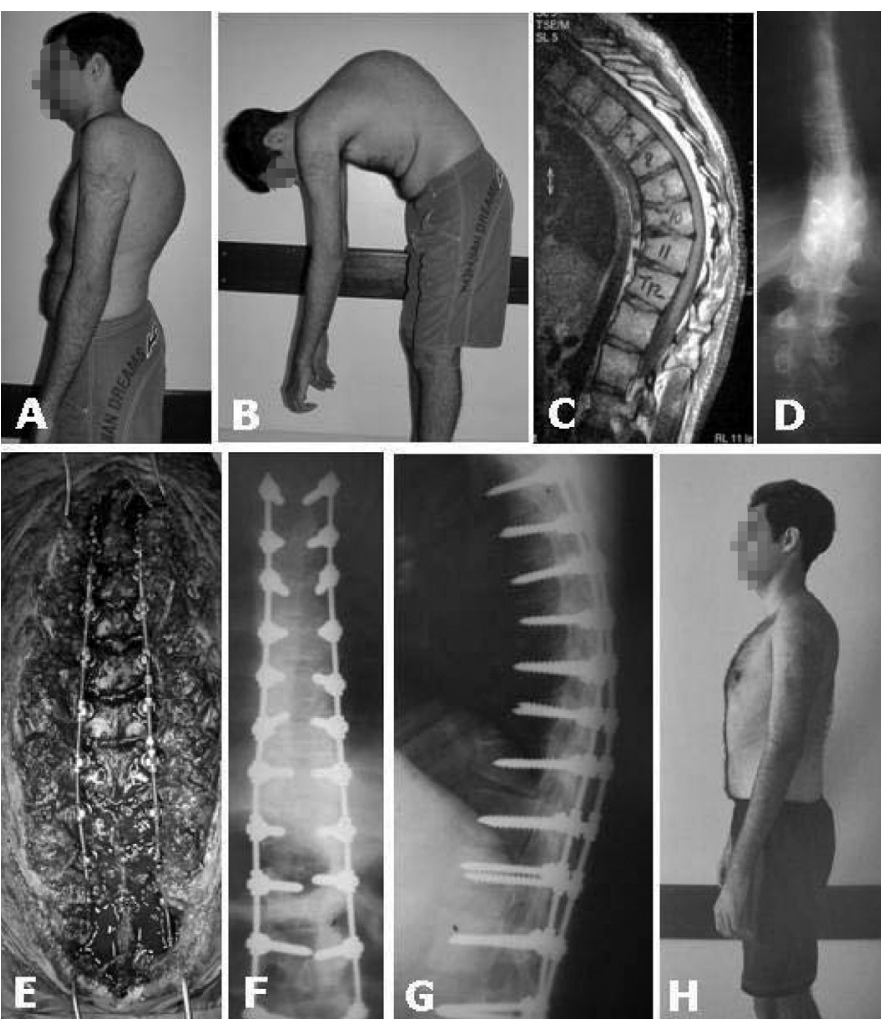

Figura 2 - Paciente no 2 da Tabela 1. Aspecto clínico (A e B) e radiológico (C e D) pré-operatório. Fotografia intraoperatória (E) ilustrando as osteotomias. Aspecto radiológico ( $F$ e G) e clínico (H) demonstrando a correção.

A artrodese vertebral foi realizada com enxerto autólogo de ilíaco em quatro pacientes e enxerto retirado do sitio cirúrgico e processos espinhosos em seis pacientes. Os níveis de artrodese e fixação vertebral foram selecionados considerando-se a primeira vértebra lordótica na parte distal e a vértebra terminal na parte proximal.
No período pós-operatório os pacientes não utilizaram imobilização gessada ou órtese, e a deambulação e o reinício das atividades foram permitidos de acordo com o alívio do quadro doloroso.

Os parâmetros radiológicos relacionados para a avaliação dos pacientes foram: a medida da cifose, lordose e, quando presente, da escoliose pelo método de Cobb; a cifose juncional proximal e distal, e as complicações relacionadas com a soltura ou quebra dos implantes. Os parâmetros foram avaliados no pré-operatório, pós-operatório imediato e avaliação tardia. Todos os pacientes foram seguidos por um período mínimo de dois anos (Tabela 1).

\section{RESULTADOS}

Os pacientes foram seguidos por um período que variou de 24 a 144 meses (16,8 $\pm 39,92$ meses).

A cifose pré-operatória variou de $72^{\circ}$ a $96^{\circ}\left(78,8^{\circ}\right.$ $\left.\pm 7,59^{\circ}\right)$, de $28^{\circ}$ a $70^{\circ}\left(44,9^{\circ} \pm 12,1^{\circ}\right)$ no período pósoperatório imediato e de $34^{\circ}$ a $72^{\circ}\left(47,5^{\circ} \pm 12,54^{\circ}\right)$ na avaliação tardia (Figura 3 ). A correção da cifose variou de $50^{\circ}$ a $21^{\circ}\left(33,9^{\circ} \pm 9,53^{\circ}\right)$ no período pré-operatório imediato e foi observada pequena perda da correção na avaliação tardia, com média de $2,2^{\circ}$. O percentual tardio de correção da cifose variou de $61,1 \%$ a $27 \%$ $(43,25 \% \pm 12,56 \%)$.

Escoliose foi observada em três pacientes, variando de $33^{\circ}$ a $22^{\circ}$ no período pré-operatório, tendo sido observada correção de $13,3^{\circ}$ (Figuras 4 e 5). A lordose pré-operatória variou de $37^{\circ}$ a $104^{\circ}\left(61,6^{\circ} \pm 18,46^{\circ}\right)$ e, no período tardio de avaliação, de $26^{\circ}$ a $74^{\circ}\left(43,3^{\circ} \pm 13,88^{\circ}\right)$.

Tabela 1 - Dados gerais dos pacientes

\begin{tabular}{|c|c|c|c|c|c|c|c|c|c|c|c|c|c|c|c|c|c|c|c|c|}
\hline \multirow{2}{*}{ Paciente } & \multirow{2}{*}{ Sexo } & \multirow{2}{*}{ idade } & \multirow{2}{*}{ Etiologia } & \multicolumn{3}{|c|}{ Cifose } & \multicolumn{3}{|c|}{ Escoliose } & \multicolumn{3}{|c|}{ Lordose } & \multirow{2}{*}{ Cifose } & \multirow{2}{*}{ Instrumentação } & \multirow{2}{*}{$\begin{array}{c}\text { Cifose } \\
\text { juncional }\end{array}$} & \multirow{2}{*}{ Material } & \multirow{2}{*}{ Cor } & \multirow{2}{*}{$\%$ Cor } & \multirow{2}{*}{$\begin{array}{l}\text { Seguimento } \\
\text { (meses) }\end{array}$} & \multirow{2}{*}{ Complicações } \\
\hline & & & & Pré & Poi & Pot & Pré & Poi & Pot & Pré & Poi & Pot & & & & & & & & \\
\hline 1 & M & 14 & $\begin{array}{r}\text { Sequela de } \\
\text { laminectomia }\end{array}$ & $80^{\circ}$ & $50^{\circ}$ & $50^{\circ}$ & $30^{\circ}$ & $10^{\circ}$ & $10^{\circ}$ & $104^{\circ}$ & $70^{\circ}$ & $74^{\circ}$ & T4-T11 & T4-L3 & Sim & USS & $30^{\circ}$ & 37,5 & 36 & Não \\
\hline 2 & M & 18 & $\begin{array}{c}\text { Doença de } \\
\text { Scheuermann }\end{array}$ & $72^{\circ}$ & $28^{\circ}$ & $34^{\circ}$ & - & - & - & $37^{\circ}$ & $30^{\circ}$ & $30^{\circ}$ & T4-T12 & T4-L2 & Não & USIS & $44^{\circ}$ & 61,1 & 84 & Não \\
\hline 3 & M & 12 & $\begin{array}{c}\text { Sequela de } \\
\text { laminectomia }\end{array}$ & $74^{\circ}$ & $52^{\circ}$ & $55^{\circ}$ & $15^{\circ}$ & 0 & 0 & $64^{\circ}$ & $40^{\circ}$ & $42^{\circ}$ & T2-T12 & T1-L4 & Não & USS & $22^{\circ}$ & 29,7 & 36 & Não \\
\hline 4 & $\mathrm{~F}$ & 20 & $\begin{array}{c}\text { Doença de } \\
\text { Scheuermann }\end{array}$ & $73^{\circ}$ & $52^{\circ}$ & $54^{\circ}$ & - & - & - & $72^{\circ}$ & $54^{\circ}$ & $54^{\circ}$ & T5-T12 & T3-L3 & Não & USS & $21^{\circ}$ & 28,7 & 64 & $\begin{array}{l}\text { Quebra de } \\
\text { haste }\end{array}$ \\
\hline 5 & M & 16 & $\begin{array}{c}\text { Doença de } \\
\text { Scheuermann }\end{array}$ & $82^{\circ}$ & $32^{\circ}$ & $34^{\circ}$ & - & - & - & $42^{\circ}$ & $32^{\circ}$ & $32^{\circ}$ & T3-T12 & T3-L2 & Não & USIS & $50^{\circ}$ & 60,9 & 144 & Infecção \\
\hline 6 & M & 17 & $\begin{array}{c}\text { Doença de } \\
\text { Scheuermann }\end{array}$ & $74^{\circ}$ & $42^{\circ}$ & $42^{\circ}$ & $33^{\circ}$ & $16^{\circ}$ & $20^{\circ}$ & $68^{\circ}$ & $45^{\circ}$ & $50^{\circ}$ & T5-T11 & T4-L3 & Não & USS & $32^{\circ}$ & 43,2 & 24 & Não \\
\hline 7 & $\mathrm{~F}$ & 19 & $\begin{array}{c}\text { Doença de } \\
\text { Scheuermann }\end{array}$ & $73^{\circ}$ & $35^{\circ}$ & $37^{\circ}$ & - & - & - & $58^{\circ}$ & $35^{\circ}$ & $38^{\circ}$ & T3-T12 & T3-L1 & Não & USIS & $38^{\circ}$ & 52 & 101 & Não \\
\hline 8 & $\mathrm{M}$ & 16 & $\begin{array}{c}\text { Doença de } \\
\text { Scheuermann }\end{array}$ & $96^{\circ}$ & $70^{\circ}$ & $72^{\circ}$ & - & - & - & $52^{\circ}$ & $26^{\circ}$ & $26^{\circ}$ & T3-T12 & T3-L2 & Não & USS & $26^{\circ}$ & 27 & 24 & Não \\
\hline 9 & $M$ & 20 & $\begin{array}{c}\text { Doença de } \\
\text { Scheuermann }\end{array}$ & $86^{\circ}$ & $45^{\circ}$ & $57^{\circ}$ & - & - & - & $62^{\circ}$ & $45^{\circ}$ & $44^{\circ}$ & T4-T12 & T3-L2 & Não & USIS & $41^{\circ}$ & 47,6 & 98 & Não \\
\hline 10 & $\mathrm{~F}$ & 18 & $\begin{array}{c}\text { Doença de } \\
\text { Scheuermann }\end{array}$ & $78^{\circ}$ & $42^{\circ}$ & $45^{\circ}$ & $22^{\circ}$ & $15^{\circ}$ & $15^{\circ}$ & $57^{\circ}$ & $40^{\circ}$ & $43^{\circ}$ & T4-T12 & T3-L2 & Não & USS & $35^{\circ}$ & 44,8 & 47 & Não \\
\hline
\end{tabular}

Legenda: M: masculino; F: feminino; Pré: pré-operatória; Poi: pós-operatória imediata; Pot: pós-operatória tardia; Cor: correção em graus; \%Cor: porcentagem da correção. 


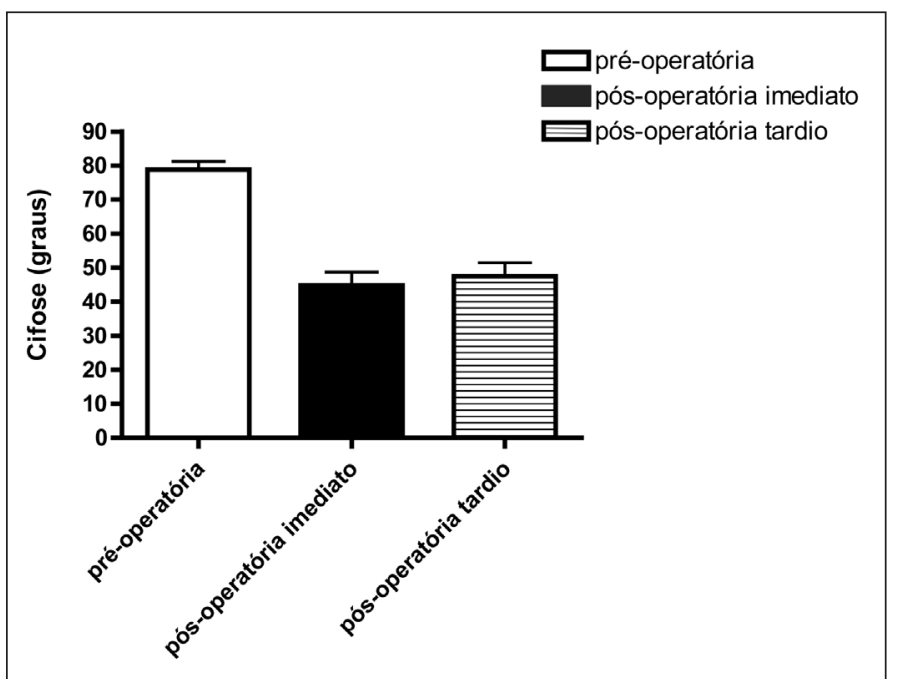

Figura 3 - Gráfico ilustrando as médias dos valores angulares da cifose nos períodos pré-operatório, pós-operatório imediato e pós-operatório tardio.
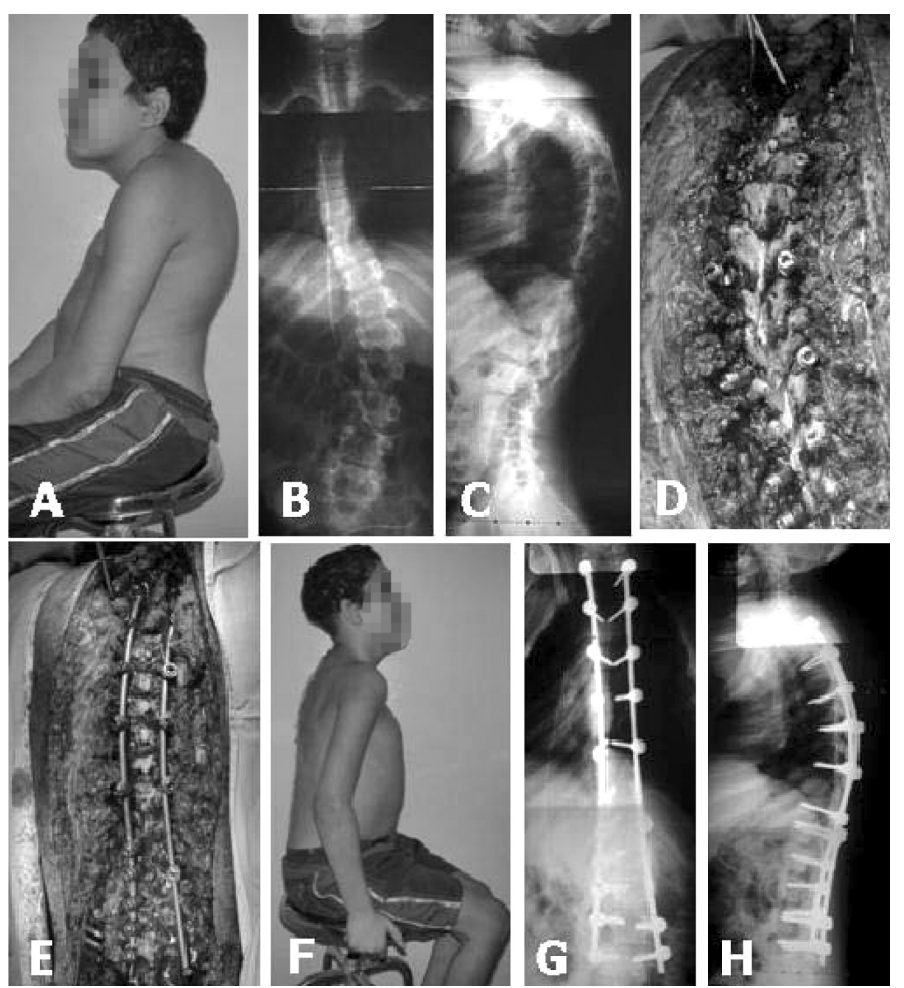

Figura 4 - Paciente $\mathrm{n}-1$ da Tabela 1. Fotografia $(\mathrm{A})$ e radiografias ( $\mathrm{B}$ e C) pré-operatórias evidenciando cifose e escoliose associada. Fotografia intraoperatória (D e E) ilustrando as osteotomias múltiplas e correção final. Aspecto clínico $(F)$ e radiográfico (G e H) demonstrando correção da cifose.

A cifose juncional proximal de $35^{\circ}$ foi observada em um paciente $\left(n^{\circ} 1\right)$, que não apresentava repercussão clínica. Esse paciente apresentava sequela de laminectomia utilizada para ressecção de astrocitoma pilocítico. A cifose juncional distal não foi observada em nenhum dos pacientes neste estudo.
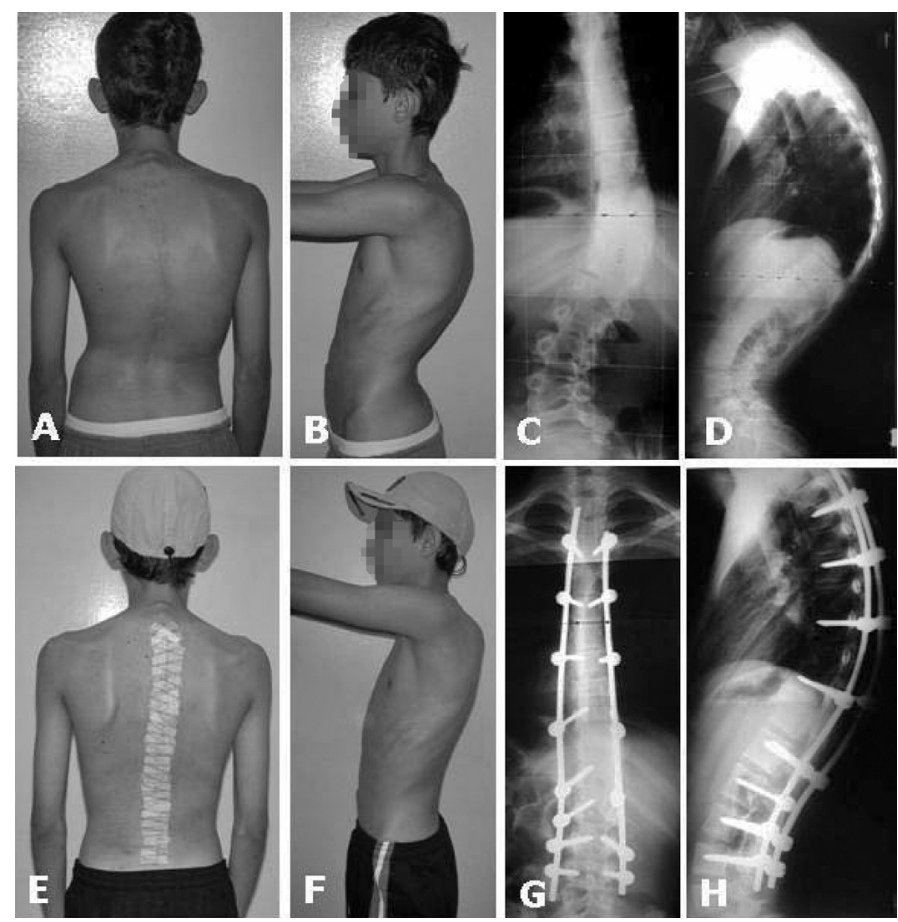

Figura 5 - Paciente $\mathrm{n}^{\circ} 3$ da Tabela 1. Fotografias (A e B) e radiografias $(C$ e $D)$ pré-operatórias evidenciando aumento da cifose e a presença de escoliose. Aspectos clínico (E e F) e radiográfico ( $G$ e H) pós-operatório demonstrando a correção das deformidades.

Infecção tardia profunda da ferida operatória foi observada após dois anos da cirurgia em um paciente $\left(\mathrm{n}^{\circ}\right.$ 5 da Tabela 1), tendo sido tratado com sucesso por meio de limpeza cirúrgica sem a retirada dos implantes. Um paciente ( $\mathrm{n}^{\circ} 4$ da Tabela 1) apresentou quebra dos implantes e foi reoperado para troca do material de fixação e complementação com enxerto ósseo, tendo sido observados no intraoperatório dois focos de pseudoartrose.

\section{DISCUSSÃO}

As indicações cirúrgicas para a correção de hipercifoses dorsais têm sido direcionadas para a correção de deformidade acima de 70 graus, deformidades progressivas, dor resistente ao tratamento conservador ou deformidade cosmética inaceitável ${ }^{(9,10,21)}$. Esses parâmetros em conjunto ou isolados norteiam o tratamento cirúrgico. Existem poucas controvérsias com relação à indicação cirúrgica. A avaliação dos resultados do tratamento é complexa, pois a dor e a alteração cosmética são parâmetros subjetivos e é difícil sua quantificação.

Os primeiros relatos cirúrgicos dessa deformidade referem-se à abordagem posterior ${ }^{(22)}$ com uso do sistema de Harrington de compressão, que apresentava alta porcentagem de perda de correção durante o seguimento $^{(15,22)}$. A abordagem de dupla via, anterior 
associada à posterior, foi preconizada para reduzir a perda da correção. Devido à melhora dos resultados, ainda é muito utilizada ${ }^{(16)}$.

Ponte e Siccardi ${ }^{(19)}$ descreveram a técnica da correção posterior isolada com ressecção posterior dos elementos vertebrais e encurtamento da convexidade da deformidade utilizando a técnica de compressão aplicada no sistema de fixação. A técnica descrita por Ponte e Siccardi é uma modificação da osteotomia de Smith-Petersen, com o contato das superfícies ósseas posteriores ${ }^{(19)}$.

Desde o início do emprego da técnica de Ponte utilizamos a fixação pedicular em todos os níveis da coluna vertebral. Nos primeiros casos foi utilizada haste rosqueada de $4 \mathrm{~mm}$ e, depois, haste não rosqueada de $6 \mathrm{~mm}$. Entretanto, cabe ressaltar que a técnica de Ponte utilizava ganchos ${ }^{(19)}$.

A correção obtida em nossa série de pacientes foi satisfatória, estando de acordo com a de outros relatos ${ }^{(10-29)}$, deve ser observado o cuidado para evitar-se a hipercorreção por meio dessa técnica.

A comparação entre a correção anterior e a posterior, com a correção posterior isolada, tem sido relatada na literatura atual ${ }^{(10-29)}$. Apesar da correção satisfatória da hipercifose dorsal por meio da abordagem posterior isolada pela técnica de Ponte, ainda não está esclarecido se essa técnica possui a mesma capacidade de correção em curvas de mesmo valor quando comparada com a abordagem combinada $^{(18,27)}$. No entanto, o objetivo da correção da deformidade nas hipercifoses não é a correção absoluta da deformidade, mas a obtenção de valores dentro do espectro da normalidade associado ao equilíbrio no plano sagital.

A manutenção da correção no seguimento tardio tem sido outro parâmetro de comparação mencionado entre as técnicas. No grupo estudado esse parâmetro foi satisfatório (média de 33, $9^{\circ}$ ). Vários relatos mostram tendência de menor perda da correção por meio da utilização da abordagem combinada $^{(18,19,27,30)}$. Essa diferença, embora estatisticamente significativa, é representada por pequenos valores: $3^{\circ}$ e $4^{\circ}$ (combinada) e $6^{\circ}$ (posterior) e deve ser interpretada com cautela. A literatura relata perda de $15^{\circ}$ após a remoção dos implantes ${ }^{(30)}$. O tipo de instrumentação posterior (parafusos pediculares, ganchos ou sistemas híbridos) deve também ser considerado na avaliação da perda de correção tardia. Na teoria, a realização da abordagem combinada promoveria a consolidação mais rápida no ápice da deformidade, preservando desse modo a correção obtida ${ }^{(14,31)}$.

Nesse grupo de pacientes, a lordose lombar apresentou redução em relação aos valores pré-operatórios, estando de acordo com a literatura ${ }^{(10)}$, que tem correlacionado a lordose com o grau de correção da cifose $\mathrm{e}^{(10,19,32)}$, apresentando aumento do seu valor no seguimento tardio ${ }^{(10,32)}$.
A lordose pós-operatória é influenciada pela cifose obtida após a correção e, desse modo, o mecanismo de compensação no plano sagital estaria relacionado com a morfologia intrínseca da pelve (incidência pélvica) e também com as alterações no alinhamento e flexibilidade da coluna impostas pela artrodese. A cifose pré-operatória não tem apresentado correlação com a incidência pélvica ${ }^{(10)}$.

O percentual de complicações tem sido menor nos pacientes submetidos à abordagem posterior, devido à não realização da toracotomia ${ }^{(18,27)}$. Em nossa série de pacientes as complicações foram a quebra de material em um caso ( $n^{\circ} 4$ da Tabela 1) e infecção de ferida operatória em outro ( $\mathrm{n}^{\mathrm{o}} 5$ da Tabela 1$)$, ambas resolvidas com o tratamento cirúrgico realizado.

A cifose juncional com repercussão clínica (proximal ou distal) não ocorreu em nosso grupo de pacientes. Apenas um paciente ( $n^{\circ} 1$ da Tabela 1$)$ apresentou evidente deformidade radiográfica de 35 graus no segmento proximal à instrumentação, mas sem queixa de deformidade clínica, não tendo sido necessária a realização de tratamento adicional.

A ocorrência de cifose juncional tem sido mais relevante do ponto de vista radiológico que clínico, embora alguns pacientes tenham sido reoperados para a resolução desse problema. A ocorrência da cifose juncional proximal tem sido correlacionada com a correção superior a $50 \%$ e instrumentação proximal curta, distal à vértebra terminal superior ${ }^{(10)}$. Na série de Lonner et $a l^{(9)}$, a ocorrência de cifose juncional proximal esteve relacionada com a magnitude pré-operatória da deformidade e com o grau de correção. No entanto, não foi possível concluir se esse fato indica a dificuldade do paciente com grandes curvas em atingir o equilíbrio sagital normal sem desequilibrar os seguimentos adjacentes à área de artrodese, ou se existiria algo relacionado com as estruturas segmentares da transição da área de artrodese e instrumentação.

Essa cifose juncional tem sido relacionada com a vértebra inferior da artrodese (LIV); tem sido recomendada a utilização de vértebra estável sagital como parâmetro da relação da LIV. Em nossa série a LIV foi distal à vértebra estável sagital em todos os casos, prevenindo em nossos pacientes o desenvolvimento de cifose juncional distal.

\section{CONCLUSÃO}

O tratamento cirúrgico da hipercifose dorsal utilizando a abordagem posterior isolada com osteotomias múltiplas e fixação com parafusos pediculares mostrouse boa opção de tratamento, alcançando valores dentro dos parâmetros fisiológicos e apresentando durante o seguimento perda mínima da correção inicial. 


\section{REFERÊNCIAS}

1. Hefti F. Morbus Scheuermann - Scheuermanns juvenile kyphosis. Ther Umsch. 1987;44(10):764-70.

2. Macagno AE, O'Brien MF. Thoracic and thoracolumbar kyphosis in adults. Spine. Spine (Phila Pa 1976). 2006;31(19 Suppl):S161-70.

3. Scheuermann H. Kyfosis dorsalis juvenilis. Ugeskr Laeger. 1920;82:385-93.

4. Aufdermaur M, Spycher M. Pathogenesis of osteochondrosis juvenilis Scheuermann. J Orthop Res. 1986;4(4):452-7.

5. Blazek O, Streda A, Cermak V, Skallova O. The incidence of morbus Scheuermann in sportsmen. J Sports Med Phys Fitness. 1986;26(1):55-9.

6. Griffet J Bastiani F. Fracture of lombar spine in child simulating Scheuermann disease. Chir Pediatr. 1987;28(4-5):259-61.

7. King JC, King JC. Scheuermann's disease: the lumbar variant. Am J Phys Med Rehabil. 2004;83(6):467.

8. Lowe TG. Current concepts review - Scheuermann disease. J Bone Joint Surg Am. 1990;72(6):940-5.

9. Lonner BS, Newton P, Betz R, Scharf C, O’Brien M, Sponseller P., et al. Operative management of Scheuermann's kyphosis in 78 patients: radiographic outcomes, complications, and technique. Spine. 2007;32(24):2644-52.

10. Bradford DS, Moe JH, Montalvo F, Winter RB. Scheuermanns juvenile kyphosis. Experience in 200 patients. J Bone Joint Surg Am. 1973;55(6):1323.

11. Bradford DS, Moe JH, Montalvo FJ, Winter RB. Scheuermanns kyphosis and roundback deformity. Results of Milwaukee brace treatment. J Bone Joint Surg Am. 1974;56(4):740-58.

12. Krismer M, Behensky H, Frischhut B, Wimmer C, Ogon M. Operative treatment of idiopathic scoliosis and juvenile kyphosis. Orthopade. 2002;31(1):26-33.

13. Winter R. The Treatment of spinal kyphosis. Int Orthop. 1991;15(3):265-71.

14. Arlet V, Schlenzka D. Scheuermann's kyphosis: surgical management. Eu Spine J. 2005;14(9):817-27.

15. Arlet V. Anterior thoracoscopic spine release in deformity surgery: a metaanalysis and review. Eur Spine J. 2000;9(Suppl 1):S17-23.

16. Berven SH, Deviren V, Smith JA, Hu SH, Bradford DS. Management of fixed sagittal plane deformity: outcome of combined anterior and posterior surgery. Spine (Phila Pa 1976). 2003;28(15):1710-5

17. Lee SS, Lenke LG, Kuklo TR, Valente L, Bridwell KH, Sides B, et al. Comparison of Scheuermann kyphosis correction by posterior-only thoracic pedicle screw fixation versus combined anterior/posterior fusion. Spine (Phila Pa 1976). 2006;31(20):2316-21.

18. Macedo RD, Fim M, Fontes BPC. Parafusos pediculares no tratamento da cifose de Scheuermann: resultados e complicações. Rev Bras Ortop. 2008;43(1/2):23-30.

19. Ponte A, Siccardi GL, Ligure P. Posterior shortening procedure by segmental closing wedge resections [Abstract]. J Pediatr Orthop. 1995;15:404

20. Webb JK. Comment to "Clinical outcome and radiographic results after operative treatment of Scheuermann's disease", by R. W. Poolman et al. Eur Spine J. 2002;11(6):570.

21. Bradford DS, Moe JH, Montalvo FJ, Winter RB. Scheuermanns kyphosis. Results of surgical treatment by posterior spine arthrodesis in 22 patients. J Bone Joint Surg Am. 1975;57(4):439-48

22. Gaines RW. Reconstrução curta para tratamento da escoliose idiopática do adolescente e doença de Scheuermann baseada na discectomia total. Coluna/ Columna. 2005;4(3):113-68.

23. Herndon WA, Emans JB, Micheli LJ, Hall JE. Combined anterior and posterior fusion for Scheuermann kyphosis. Spine (Phila Pa 1976). 1981;6(2):125-30.

24. de Jonge T, Illes T, Bellyei A. Surgical correction of Scheuermann's kyphosis. Int Orthop. 2001;25(2):70-3.

25. Gertzbein SD, Harris MB. Wedge osteotomy for the correction of posttraumatic kyphosis. A new technique and a report of 3 cases. Spine (Phila Pa 1976). 1992;17(3):374-9.

26. Johnston CE, Elerson E, Dagher G. Correction of adolescent hyperkyphosis with posterior-only threaded rod compression instrumentation: Is anterior spinal fusion still necessary? Spine (Phila Pa 1976). 2005;30(13):1528-34.

27. Lehmer SM, Keppler L, Biscup RS, Enker P, Miller SD, Steffee AD. Posterior transvertebral osteotomy for adult thoracolumbar kyphosis. Spine (Phila Pa 1976). 1994;19(18):2060-7.

28. Saraph VJ, Bach CM, Krismer M, Wimmer C. Evaluation of spinal fusion using autologous anterior strut grafts and posterior instrumentation for thoracic/thoracolumbar kyphosis. Spine (Phila Pa 1976). 2005;30(14):1594-601.

29. Poolman RW, Been HD, Ubags LH. Clinical outcome and radiographic results after operative treatment of Scheuermann's disease. Eur Spine J. 2002;11(6):561-9.

30. Hosman AJF. Letter to the Editor regarding "Scheuermann's kyphosis: surgical management” (V. Arlet, D. Schlenzka). Eur Spine J. 2006;15(9):1411-2.

31. Papagelopoulos PJ, Klassen RA, Peterson HA, Dekutoski MB. Surgical treatment of Scheuermann's disease with segmental compression instrumentation. Clin Orthop Relat Res. 2001;(386):139-49.

32. Lim M, Green DW, Billinghurst JE, Huang RC, Rawlins BA, Widmann RF, et al. Scheuermann kyphosis: safe and effective surgical treatment using multisegmental instrumentation. Spine (Phila Pa 1976). 2004;29(16):1789-94. 